\title{
La mère et l'enfant atteints de dystrophie myotonique de Steinert
}

\author{
G. Paris, R. Laframboise et J.-P. Bouchard
}

\begin{abstract}
RÉSUMÉ: La grossesse et l'accouchement présentent des dangers particuliers pour la femme atteinte de dystrophie myotonique de Steinert. Il arrive assez souvent que la mère, encore jeune et pas toujours très symptomatique, ignore d'ailleurs qu'elle est atteinte et porteuse de cette maladie. Nous rappelons ici les complications de la grossesse associées à la dystrophie myotonique de Steinert et proposons une conduite à tenir vis-à-vis de la mère. Quant à l'enfant né de mère dystrophique, non seulement présente-t-il un risque de 50\% d'hériter du gène de la maladie, mais il peut aussi présenter un s̀yndrome développemental et malformatif particulier. Aux difficultés respiratoires des premières heures succèdent des troubles de déglutition, une hypotonie sévère et plus tard un retard psychomoteur important. Nous illustrons quelques-unes des manifestations retrouvées chez le nouveau-né et le nourrisson atteint de dystrophie myotonique congénitale et nous ajoutons quelques tests pour compléter le bilan habituellement recommandé chez ces patients.
\end{abstract}

\begin{abstract}
The Mother and Infant with Myotonic Dystrophy Pregnancy and delivery present a number of risks for the mother suffering from myotonic dystrophy, and for her infant. Most of the time, she does not even know that she is affected by the disease and a carrier of the gene. We review the complications of pregnancy and delivery in myotonic patients, and propose a simple management with specific items for each gestational period. The child of a dystrophic mother has a $50 \%$ risk of inheriting the abnormal gene. He may also exhibit a developmental and malformation syndrome called "congenital myotonic dystrophy". From the beginning, he may show respiratory distress, thereafter inability to swallow and severely hypotonia. Later, he may demonstrate mental retardation. Some of the most obvious signs found in neonates in our practice are illustrated. We also add a few tests to the list of those already recommended for these children.
\end{abstract}

Can.J. Neurol.Sci. 1989; 16: 104-108

La première description de la dystrophie myotonique de Steinert (DMS), qui porte son nom par Steinert en 1909, ne faisait aucune mention de la nature héréditaire du problème, et encore moins de complications gynécologiques, obstétricales ou néonatales maintenant reconnues comme associées à cette maladie. Ce n'est d'ailleurs pas avant 1940 que Mass ${ }^{1}$ rapporte une aggravation de la myotonie en période menstruelle ou gestationnelle avec amélioration en post-partum, une observation qui ne semble pas avoir été confirmée par la suite. En 1948, Thomasen ${ }^{2}$ note pour sa part une augmentation de l'irrégularité menstruelle en fonction de l'atteinte musculaire. Sciarra ${ }^{3}$ rapporte en 1961 une observation qui suggère une prolongation de la phase de relaxation utérine. En 1971, Shore ${ }^{4}$ résume l'évolution de la grossesse chez huit patientes dystrophiques; il ajoute alors deux cas personnels aux six autres publiés entre 1958 et 1969. Il souligne une augmentation du taux d'avortement, du taux de complications obstétricales et du taux de mortalité néonatale.

Il faut attendre 1975 pour qu'apparaisse une étude majeure, celle de Harper, ${ }^{5}$ qui présente l'histoire gynécologique et obstétricale de 44 patientes dystrophiques. Plus près de nous, Webb en $1978^{6}$ rapporte 23 grossesses chez six femmes dystrophiques au Labrador.

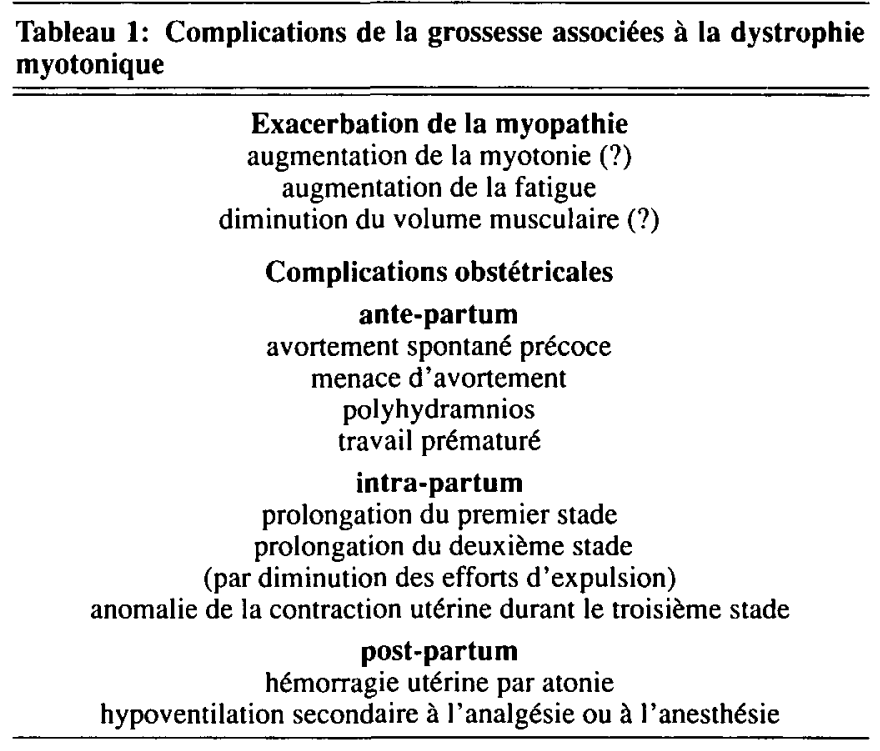

Les anomalies du muscle strié dans la DMS sont bien connues mais encore mal comprises. On retrouve aussi une atteinte fréquente des muscles lisses au niveau du pharynx, de

Département de gynéco-obstétrique (GP) et Département des sciences neurologiques (J.-P.B), Hôpital de l'Enfant-Jésus, Université Laval, Québec et Service de médecine génétique (R.L.), Centre hospitalier de l'Université Laval, Sainte- Foy

Tirés à part: Dr. Gaétan Paris, Département de gynéco-obstétrique, Hôpital de l'Enfant-Jésus, 1401, 18ième Rue, Québec, Canada GlJ 1 Z4 
l'oesophage, du diaphragme et de l'intestin. Par exemple, la dysphagie est beaucoup plus fréquente que les malades ne l'avouent (2/3), provoquant parfois des aspirations pulmonaires. Quant à l'utérus, son atteinte n'a jamais été clairement démontrée en pathologie, mais sa fonction paraît souvent perturbée comme nous le verrons plus loin.

La DMS est souvent peu évidente et non diagnostiquée chez les jeunes adultes, et même parfois tardivement dans son évolution. Pour certains cas, le diagnostic repose uniquement sur un index élevé de suspicion. Dans ces circonstances, on peut comprendre que plusieurs cas de DMS chez la femme soient reconnus seulement à la suite d'une complication obstétricale ou néonatale. ${ }^{7}$

\section{Complications de la grossesse associées à la dystrophie myotonique}

Shore ${ }^{8}$ en 1975, dans un tableau basé sur une revue de la littérature, présente ainsi les complications de la grossesse associée à la DMS (Tableau 1).

Durant la grossesse, le premier trimestre est marqué par une augmentation significative du taux d'avortement. Harper ${ }^{5}$ évalue ce taux à deux ou trois fois la normale. Au dernier trimestre, Shore ${ }^{8}$ mentionne une augmentation du travail prématuré, ce qui n'est pas retrouvé dans l'étude de Harper.

L'hydramnios represente une complication majeure de la grossesse car il est généralement signe d'une atteinte foetale. L'hydramnios est causé par une déglutition anormale du foetus, tel que démontré par Dunn ${ }^{9}$ en 1973 au moyen d'Hypaque ${ }^{\circledR}$ intra-amniotique. Broekhuisen ${ }^{10}$ propose aussi en 1983 un autre facteur pouvant contribuer à l'hydramnios, soit une diminution de la réabsorption pulmonaire du liquide amniotique, secondaire à une diminution des mouvements respiratoires intra-utérins. On retient cependant que le taux de grossesse compliquée d'hydramnios dans la DMS n'est généralement pas augmenté

\section{Tableau 2: Conduite vis-à-vis de la mère atteinte de DMS}

\section{- Conseil génétique}

- Surveillance de l'activité foetale

- Surveillance échographique

$$
\text { - activité foetale }
$$

- hydramnios

- présentation

- localisation placentaire

- malformations squelettiques

- Evaluation respiratoire de la patiente en prévision de

l'accouchement ou de toute forme d'anesthésie — inhalothérapie

- physiothérapie respiratoire

- consultation en anesthésie

- Prolongation possible du deuxième stade du travail - considérer l'utilisation d'une ventouse

- Possibilité accrue d'hémorragie du post-partum - sang en réserve - oxytociques

- Possibilité d'une réanimation néonatale intensive

lorsque le foetus est génétiquement normal ou que la transmission est paternelle. Il faut donc que la mère et le foetus soient atteints pour que l'on retrouve un hydramnios, et dans ce cas le foetus est également hypoactif.

L'échographie obstétricale permet de noter une diminution des mouvements foetaux et des mouvements respiratoires, une fréquence plus élevée de présentation du siège, et certaines anomalies squelettiques comme les pieds bots. De plus, l'absence d'image de l'estomac peut suggérer une déglutition déficiente. Il n'est pas rare, sur une radiographie du contenu utérin, de voir un enfant la bouche ouverte, signalant déjà une atonie des masséters."

Dans la période intra-partum, certains auteurs comme Sciarra ${ }^{3}$ et Shore $^{4}$ ont noté des anomalies de la contraction utérine prolongeant le premier stade du travail. Mais c'est dans

Tableau 3: Steinert congénital CHUL 1979-1987

\begin{tabular}{|c|c|c|c|c|}
\hline Sexe & $\begin{array}{l}\# 1 \\
\mathbf{M} \\
\end{array}$ & $\begin{array}{l}\# 2 \\
\mathbf{M} \\
\end{array}$ & $\begin{array}{l}\# 3 \\
\mathbf{F}\end{array}$ & $\begin{array}{l}\# 4 \\
\mathrm{M}\end{array}$ \\
\hline $\begin{array}{l}\text { P.N./taille/P.C. } \\
(\mathrm{kg})(\mathrm{cm})(\mathrm{cm})\end{array}$ & $3.100 /-/ 37.5$ & $2.840 / 51 / 38.5$ & $3.750 / 55 / 36.5$ & $3.700 /-/ 38.5$ \\
\hline \multicolumn{5}{|l|}{ Grossesse } \\
\hline Gestation (sem.) & 38 & 39 & 40 & 40 \\
\hline Mouv. foetaux & peu & peu & peu & peu \\
\hline Hydramnios & + & + & + & + \\
\hline Acc.: siège & + & + & + & + \\
\hline : césarienne & + & + & + & + \\
\hline \multicolumn{5}{|l|}{ Clinique } \\
\hline $\begin{array}{l}\text { Hypotonie } \\
\text { (attitude batracien) }\end{array}$ & Sévère & Sévère & Sévère & Sévère \\
\hline Détresse respiratoire & + & + & + & + \\
\hline Intubation & + & + & + & + \\
\hline Décès & $(6$ ans $11 / 12)$ & $\stackrel{+}{(16 \text { mois })}$ & $\stackrel{+}{+}\left(20_{\text {jours }}\right)$ & $\stackrel{+}{+}$ \\
\hline \multicolumn{5}{|l|}{ Dysmorphie } \\
\hline Macrocranie & + & + & + & + \\
\hline Lèvre supérieure en tente & + & + & + & + \\
\hline Palais ogival & + & + & + & + \\
\hline Rétrognathie & + & + & + & + \\
\hline Pieds: équinovarus & + & + & - & + \\
\hline Contractures & + & + & - & + \\
\hline Transmission génétique & mère & mère & mè̀re & mère \\
\hline
\end{tabular}




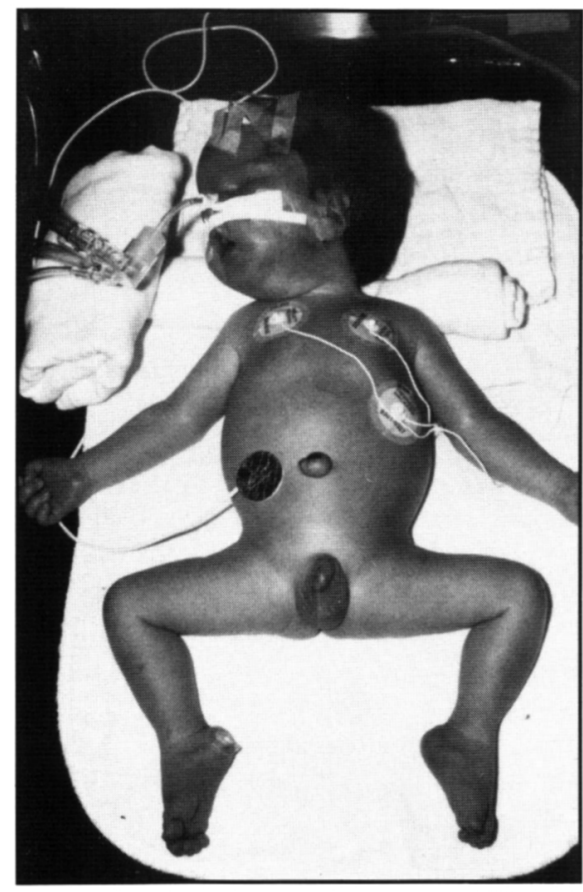

Figure l-Nouveau-né atteint de DMS, âgé de 24 heures.

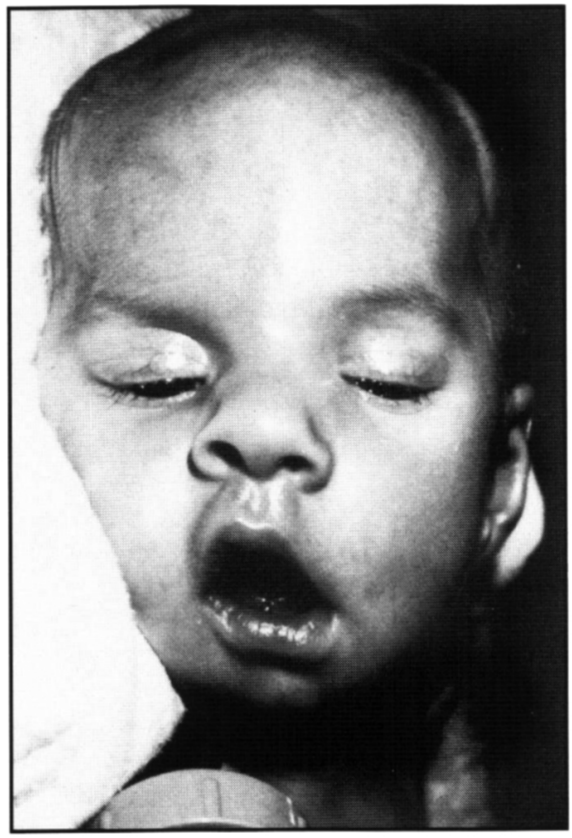

Figure 2 - Nourisson (DMS) âgé de 7 semaines avec une diplégie faciale.

le deuxième stade du travail que tous les auteurs s'accordent pour dire qu'il existe une diminution des efforts volontaires d'expulsion. Cette prolongation du travail est parfois accompagnée de souffrance ou de mort foetale, et nécessite une intervention à l'aide d'un forceps. Dans le troisième stade du travail, I'hémorragie représente un risque significatif. L'atonie utérine et la rétention placentaire en sont responsables. On a aussi rapporté une incidence accrue de placenta praevia et accreta.

Du point de vue anesthésique, l'atteinte du pharynx et de l'oesophage, provoquant le relâchement du sphincter cricopharyngé, augmente le risque d'aspiration trachéale. On note également des périodes d'hypoventilation en post-partum d'accouchements par ailleurs normaux ou de césariennes. A ce titre, les patientes dystrophiques sont très sensibles aux analgésiques et aux agents anesthésiques.

La mortalité néonatale est plus importante que la mortalité foetale. Selon Harper ${ }^{5}$ elle atteint $160 / 1000$ naissances comparativement à 19/1000 pour une même période. Cet excès de mortalité néonatale serait expliqué en grande partie par la défaillance respiratoire des enfants affectés, soit par une atteinte directe du diaphragme et des muscles intercostaux, soit par immaturité pulmonaire, ou encore par pneumonie d'aspiration.

Le grand nombre d'anomalies notées au cours de la grossesse et de l'accouchement des femmes atteintes de DMS suggère une conduite appropriée telle que résumée dans le Tableau 2. Quant au phénotype clinique des nouveaux-nés atteints de DMS, maintenant bien connu, il fait l'objet de la deuxième partie de ce travail.

\section{La dystrophie myotonique congénitale}

La forme congénitale ou néonatale de la DMS diffère cliniquement de la forme adulte. ${ }^{12-13}$ Elle peut se manifester à notre avis de deux façons: soit sous une forme sévère avec malformations congénitales et détresse respiratoire importante, forme souvent léthale dans les premiers jours ou premiers mois de vie; sois sous une autre forme, beaucoup plus difficile à reconnaître à la naissance, faite d'hypotonie fruste et de problèmes de déglutition au début, évoluant ensuite vers un retard de développement neuromoteur et une déficience mentale. Dans la littérature française, cette dernière forme est appelée par certains ${ }^{15}$ forme infantile précoce.

On voit plus souvent la première forme que la seconde dans les centres tertiaires de pédiatrie ou les unités de soins intensifs pour nouveaux-nés et notre expérience le confirme (Tableau 3). La forme congénitale diffère également de la forme adulte de par sa transmission maternelle probablement exclusive. Rappelons aussi que l'atteinte maternelle n'est souvent trouvée qu'à posteriori, suite au diagnostic de DMS chez le nouveau-né.

Le phénotype clinique de la forme congénitale de la DMS a déjà fait l'objet de nombreuses publications. ${ }^{14-19}$ Tous les signes décrits pourraient globalement s'expliquer par l'hypotonie et l'akinésie foetales.

Le tableau classique de la forme congénitale sévère se présente comme suit: une femme jeune dont la grossesse s'est caractérisée par un polyhydramnios à partir de la 30 ième semaine gestationnelle, donne naissance à un enfant peu réactif dont la détresse respiratoire nécessite une intubation dans les premières heures de vie. Malgré un âge gestationnel adéquat et un poids de naissance approprié, l'enfant a des problèmes de succion et de déglutition. L'apparence extérieure révèle un enfant plutôt longiligne et marfanoïde, avec parfois des contractures aux coudes et aux genoux, de même qu'un flexum de la hanche. Les extrémités démontrent une arachnodactylie et des pieds bots, tous ces signes étant secondaires au peu de mouvement intra-utérin (Figure 1).

Le faciès est étroit et présente une hypotrophie des fosses temporales et des signes de diplégie faciale (Figure 2): une bouche entrouverte et une lèvre supérieure «en tente», un palais ogival dont l'étroitesse peut aller jusqu'à la fente, et une 


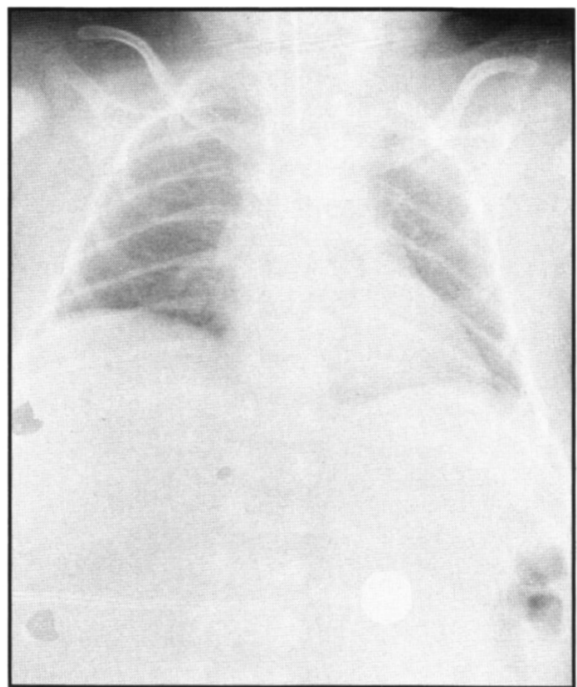

Figure 3 - Radiographie pulmonaire d'un nouveau-né (DMS): étroitesse du tiers moyen des arcs postérieurs des côtes et surélévation de l'hémidiaphragme droit.

Tableau 4: Conditions associées à des côtes filiformes chez le nouveau-né

Dystrophie myotonique congénitale

Myopathies congénitales

Ostéogénèse impartaite

Hypothyrö̈die congénitale

Trisomie 13

Trisomie 18

Prématurité sévère

Syndrome de Hallerman-Streiff

Syndrome de Cockayne

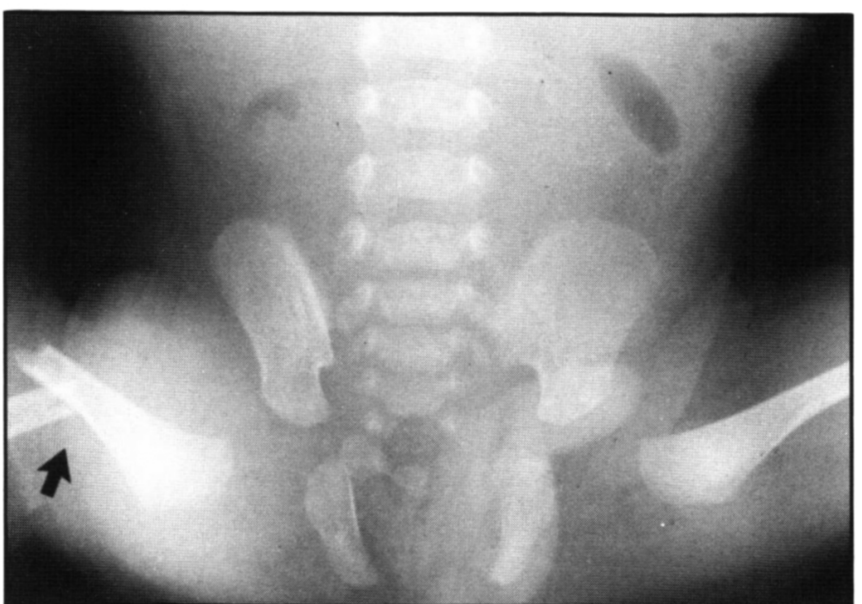

Figure 4-Radiographie du bassin: fracture au 1/3 supérieur du fémur droit.

micrognathie. Chez ces jeunes patients on note aussi une hypotonie sévère, mais les réflexes ostéo-tendineux sont habituellement préservés. On ne peut pas mettre en évidence de myotonie de percussion et l'électromyogramme est non spécifique, ne montrant pas non plus de myotonie électrique.

Du côté des examens radiologiques ${ }^{21-24}$ l'étroitesse des côtes sur la radiographie pulmonaire est frappante et assez constante
(Figure 3); elle témoigne d'un manque de tension des muscles intercostaux in utero, mais peut également se retrouver dans d'autres conditions néonatales (Tableau 4). On a aussi noté une surélévation de l'hémidiaphragme droit. Quant à nous, nous avons observé une fracture du fémur gauche chez un cas (Figure 4), et une double fracture du fémur droit et du genou gauche (Salter II) chez un autre cas. Chez ces nouveaux-nés, l'accouchement ne peut être considéré comme traumatique malgré une présentation du siège, car ces enfants furent accouchés par césarienne. Par ailleurs, le bilan phosphocalcique s'est avéré entièrement normal. ${ }^{25}$ A la suite de ces observations, nous recommandons donc d'ajouter au bilan neuromusculaire et génétique des nouveaux-nés suspects de DMS des radiographies de tout le squelette.

La forme infantile de DMS quant à elle, est plus souvent diagnostiquée lors d'un retard de développement neuromoteur, un retard de langage ou simplement par l'observation d'hypotonie. Quelques auteurs ont rapporté des phénomènes myotoniques précoces au niveau des mains ou de la langue, mais ceci est beaucoup plus rare. En rétrospective on retrouve fréquemment chez ces enfants une histoire de trouble de succion et de déglutition à la naissance. On peut donc avancer l'hypothèse d'une forme congénitale dont l'expressivité varierait de sévère à fruste (comme dans la DMS adulte), au lieu de parler de forme infantile.

\section{REMERCIEMENTS}

Nous remercions pour leur collaboration les Drs M. Masson, C. Carrier et J. Pichette, néonatologistes, et le Dr. P. Langevin, neuropédiatre, du Département de pédiatrie ainsi que le Dr Richard Gagné, généticien, du Service de médecine génétique au Centre Hospitalier de l'Université Laval (CHUL), Sainte-Foy, Québec.

\section{RÉFÉRENCES}

1. Mass O. Observations on dystrophia myotonia. Brain 1937;60: 498-524.

2. Thomasen E. Myotonia. Universitetsforlaget Aarhus, Copenhagen, 1948.

3. Sciarra JJ, Steer CM. Uterine contractions during labor in myotonic muscular dystrophy. Am J Obst Gynecol 1961; 82: 612-615.

4. Shore RN, MacLachlan TB. Pregnancy with myotonic dystrophy. Course, complications and management. Obstet Gynecol 1971; 38: 448-454.

5. Harper PS. Endocrine abnormalities in myotonic dystrophy. In: Myotonic Dystrophy. Philadelphia: WB Saunders Co, 1979.

6. Webb D. Myotonia dystrophica: obstetric complications. Am J Obst Gynecol 1978; 132: 265-270.

7. Fossen D. Obstetric complications as the first sign of myotonic dystrophy. Acta Obstet Gynecol Scand 1986; 65: 667-668.

8. Shore RN. Myotonic dystrophy: hazards of pregnancy and infancy. Dev Med Child Neurol 1975; 17: 356-361.

9. Dunn LJ, Dierker LJ. Recurrent hydramnios in association with myotonia dystrophica. Obstet Gynecol 1973; 42: 104-106.

10. Broekuizen F. Neonatal myotonic dystrophy as a cause of hydramnios and neonatal death. J Reprod Med 1983; 28: 595-599.

11. Monnier JC. Maladie de Steinert et grossesse. J Gynécol Obstet Biol Reprod 1984; 13: 541-548.

12. Vanier TM. Dystrophia myotonica in childhood. Brit Med J 1960; 2: 1284 .

13. Bell DM, Smith DW. Myotonic dystrophy in the neonate. J Pediatr 1972; 81:83-86.

14. Harper PS. Congenital myotonic dystrophy in Britain: I. Clinical aspects. Arch Dis Child 1975; 50: 505-513.

15. Tome FMS, Fardeau M. La dystrophie myotonique de Steinert: Comparaison des aspects cliniques et morphologiques rencon- 
trés chez l'adulte et chez l'enfant. G M de France 1974; 81 : 3247-3250.

16. Mundler F. Formes néonatale et infantile précoce de la dystrophie myotonique (Maladie de Steinert). Pédiatrie 1970; XXV: 865872.

17. L'hirondel J, Guihard J, Velot-Lerou A, Orange C. La maladie de Steinert néonatale. A propos d'une observation. Sem Hôp. Paris 1970; 46:1867-1874.

18. Swift TR, Ignacio OJ, Dyken PR. Neonatal dystrophia myotonica. Am J Dis Child 1975; 129: 734-737.

19. Sarnat HB, O'Connor T, Byrme PA. Clinical effect of myotonic dystrophy on pregnancy and the neonate. Arch Neurol 1976; 33: 459-465.

20. Billeaud C, Baronnet R, Cadier L, et al. Détresse respiratoire chez le nouveau-né atteint de maladie de Steinert. Méd Infant 1982; 1: 35-49.
21. Fried K, Pajewski M, Mundel G, et al. Thin ribs in neonatal myotonic dystrophy. Clin Genet 1975; 7: 417-420.

22. Chassevent J, Sauvegrain J, Besson-Leaud M, Kalifa G. Myotonic dystrophy (Steinert's disease) in the neonate. Ped Radiol 1978; 127: 747-749.

23. Chudley AE, Barmada MA. Diaphragmatic elevation in neonatal myotonic dystrophy. Am J Dis Child 1979; 133: 1182-1185.

24. Osborne JP, Murphy EG, Hill A. Thin ribs on chest X-ray: a usefull sign in the differential diagnosis of the floppy newborn. Dev Med Child Neurol 1983; 25: 343-345.

25. Laframboise R, Gagné R, Carrier C, et al. Fracture du fémur chez deux cas de dystrophie myotonique de Steinert. M/S Suppl 9 1987; 3: 25. 